\title{
Spherical Wave Near-Field Imaging and Radar Cross-Section Measurement
}

\author{
Antoni Broquetas, Member, IEEE, Josep Palau, Luis Jofre, Member, IEEE, and Angel Cardama, Member, IEEE
}

\begin{abstract}
The paper presents a new inverse synthetic aperture radar (ISAR) algorithm intended for radar cross-section (RCS) imaging and measurement from scattered fields. The method, based on a spherical-wave near-field illumination of the target, overcomes the requirement for an expensive compact range facility to produce a plane wave illumination. The formulation and the implementation of the algorithm are described. Some experimental results obtained in an anechoic chamber are presented to show RCS results similar to the conventional plane wave methods.
\end{abstract}

\section{INTRODUCTION}

A radar target is characterized by its radar cross section (RCS), which, for a given object and polarization set, depends on both the frequency and the observation angle. For analysis and diagnostics, inverse synthetic aperture radar (ISAR) techniques [1] are usually applied to the RCS data to obtain high-resolution images of the target radar reflectivity spatial distribution. The RCS is obtained by illuminating the radar target with a uniform plane wavefront and measuring the scattered fields [2]; for high frequencies or large targets, however, the plane wave illumination becomes difficult to produce with the required accuracy.

The RCS can be measured in a far-field range requiring a long distance between target and antennas. Alternatively, a compact range in an anechoic chamber requires a high-quality reflector and suffers from edge diffraction which limits the size of the quite zone (volume where the illumination can be considered a plane wave).

To overcome these problems, an alternative procedure based on near-field measurements using spherical wavefronts from low-directivity antennas has been developed.

This new algorithm consists of two parts: 1) high-resolution spherical wave imaging, which obtains the radar reflectivity image of the target from the scattered measurements using a spherical wave near-field illumination [3] and 2) RCS calculation and calibration using the known relationship between reflectivity and RCS in conventional ISAR techniques [4].

Manuscript received January 29, 1998. This work was supported by the Spanish Science and Technology Interministerial Commission (CICYT TIC 92-645).

The authors are with the Departmento de Teoría de la Señal y Comunicaciones, Grupo AMR, ETS Ingenieros Telecomunicación Barcelona, UPC, Barcelona 08080, Spain.

Publisher Item Identifier S 0018-926X(98)03422-X.

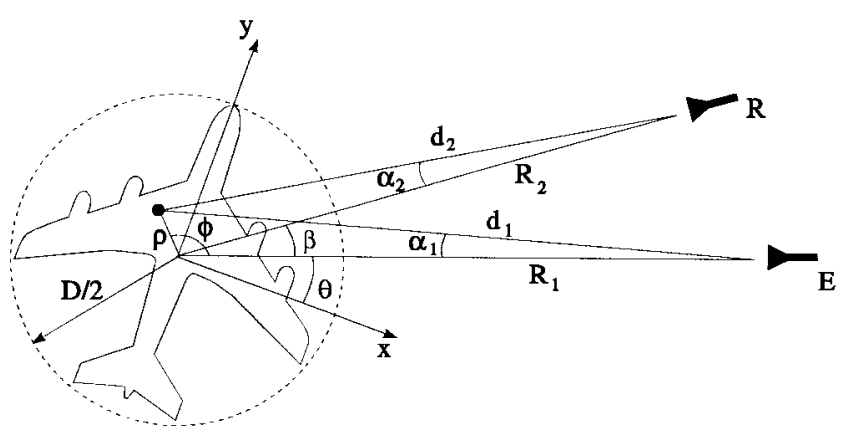

Fig. 1. Geometrical parameters of the stated problem.

\section{Problem Statement}

Consider the setup illustrated in Fig. 1 in which an object of maximum dimension $D$ (which is assumed to be planar and contained in the $z=0$ plane) with coordinates $(x, y)$ and formed by a distribution of independent and nondirectional scatterers of reflectivity $\psi(\rho, \phi)$ is rotated by a scan angle $\theta$ with respect the origin.

An emitter antenna $(E)$, located at a distance $R_{1}$ from the rotation center and with a radiation pattern $G_{E}(\alpha)$, radiates an electromagnetic field that illuminates the object.

The scattered fields $E_{S}$ are measured by a receiver antenna $(R)$ with a radiation pattern $G_{R}(\alpha)$ and located at a distance $R_{2}$ from the center, but separated by bistatic angle $\beta$ from the emitter.

Integrating the contribution of each scatterer we obtain

$$
\begin{aligned}
E_{S}(f, \theta)= & \int_{0}^{\infty} \int_{0}^{2 \pi} C \cdot \psi(\rho, \phi) \frac{e^{-j \frac{2 \pi}{\lambda}\left(d_{1}+d_{2}\right)}}{d_{1} \cdot d_{2}} \\
& \times G_{E}\left(\alpha_{1}\right) \cdot G_{R}\left(\alpha_{2}\right) \cdot \rho d \rho d \phi
\end{aligned}
$$

where $C$ is a constant depending on the parameters of the system that can be removed with calibration and where the distances from the emitter and receiver antennas to each scatterer $\left(d_{1}, d_{2}\right)$ and the illumination angles $\left(\alpha_{1}, \alpha_{2}\right)$ can be easily obtained from the geometry

$$
\begin{aligned}
d_{1} & =\sqrt{R_{1}^{2}+\rho^{2}-2 \cdot R_{1} \cdot \rho \cdot \cos (\phi-\theta)} \\
d_{2} & =\sqrt{R_{2}^{2}+\rho^{2}-2 \cdot R_{2} \cdot \rho \cdot \cos (\phi-\theta-\beta)} \\
\alpha_{1} & =\arcsin \left(\frac{\rho \cdot \sin (\phi-\theta)}{d_{1}}\right) \\
\alpha_{2} & =\arcsin \left(\frac{\rho \cdot \sin (\phi-\theta-\beta)}{d_{2}}\right) .
\end{aligned}
$$




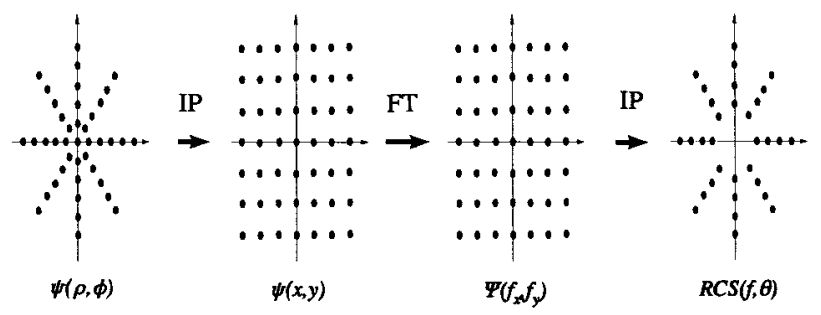

Fig. 2. Procedure to obtain RCS from reflectivity image.

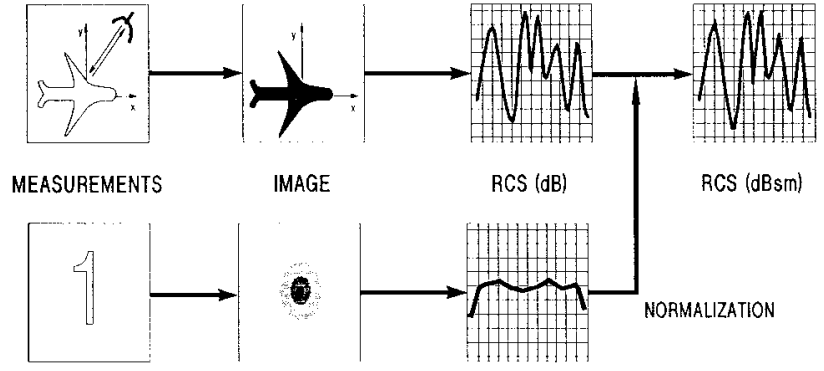

Fig. 3. RCS obtention from near-field scattering measurements.

Note that if $R_{1}, R_{2} \gg \rho$ and $\beta=0$, the plane wave monostatic case is recovered as should be expected since

$$
\begin{aligned}
R_{1} & =R_{2}=R \\
\alpha_{1} & =\alpha_{2}=\alpha \\
d_{1} & =d_{2} \\
d_{1}+d_{2} & \rightarrow 2 \cdot(R-\rho \cdot \cos (\phi-\theta)) \\
d_{1} \cdot d_{2} & \rightarrow R^{2} .
\end{aligned}
$$

Under these conditions, $E_{S}(f, \theta)$ can be expressed as a bidimensional Fourier transform of the reflectivity distribution.

\section{SPHERICAL WAVE IMAGING}

Imaging the target's radar reflectivity is a useful tool for identifying scattering mechanisms. The scattered fields $E_{S}$ can be measured over a range of frequency and azimuth and the reflectivity image $\psi$ can then be obtained by coherently focusing the field given in (1) on the image domain.

In mathematical terms

$$
\psi(\rho, \phi)=\int_{0}^{\infty} \int_{0}^{2 \pi} E_{s}(f, \theta) \cdot \xi(f, \theta, \rho, \phi) d f d \theta .
$$

The reflectivity image is obtained by applying a focusing operator $\xi(f, \theta, \rho, \phi)$ to each scattered field measurement for a given frequency and scan angle $(f, \theta)$, which restores changes incurred by the amplitude and argument of the wave on its way to and from the scatterer located at the position $(\rho, \phi)$.

Accordingly, the expression for the focusing operator is

$$
\xi(f, \theta, \rho, \phi)=\frac{e^{j \frac{2 \pi}{\lambda}\left(d_{1}+d_{2}\right)} \cdot d_{1} d_{2}}{G_{E}\left(\alpha_{1}\right) \cdot G_{R}\left(\alpha_{2}\right)} \cdot f
$$

Then we have

$\psi(\rho, \phi)=\int_{0}^{\infty} \int_{0}^{2 \pi} E_{s}(f, \theta) \cdot \frac{e^{j \frac{2 \pi}{\lambda}\left(d_{1}+d_{2}\right)} \cdot d_{1} d_{2}}{G_{E}\left(\alpha_{1}\right) \cdot G_{R}\left(\alpha_{2}\right)} \cdot f d f d \theta$

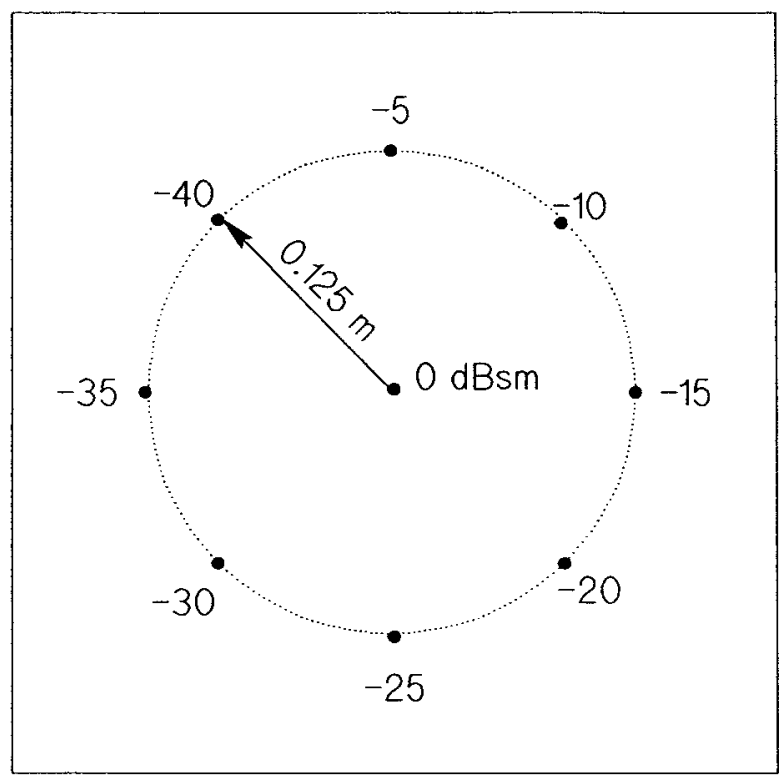

(a)

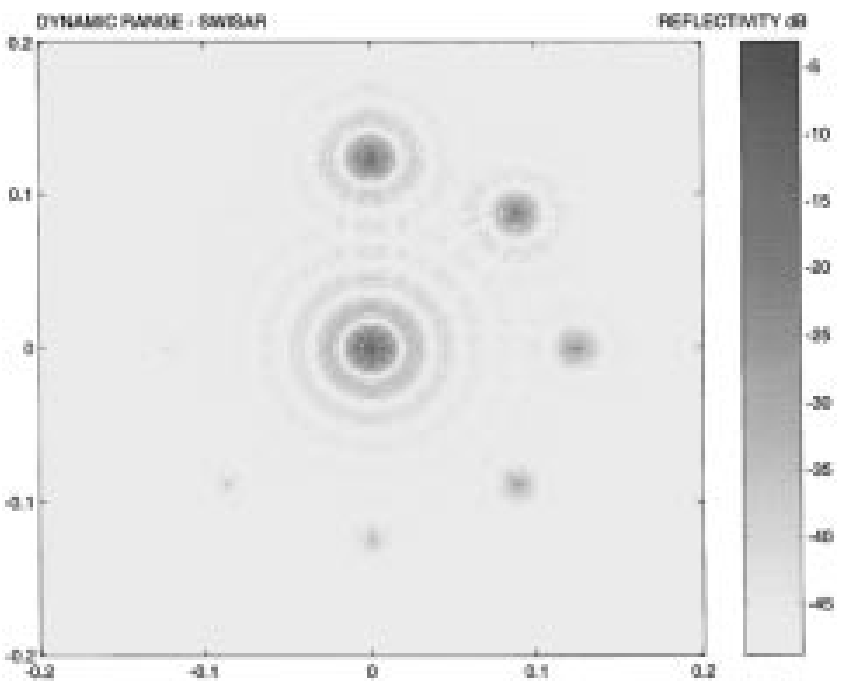

(b)

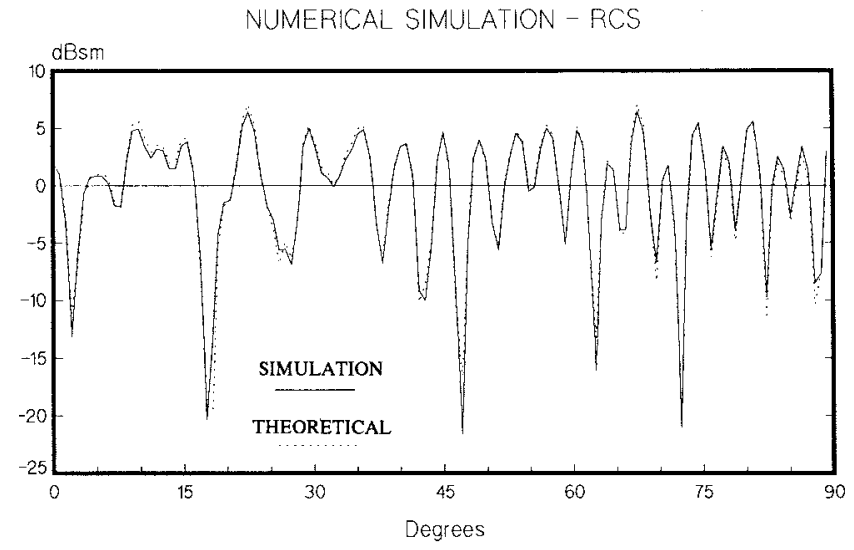

(c)

Fig. 4. (a) Target modeled to evaluate the dynamic range. (b) Reflectivity image of the modeled target. (c) RCS curves of the modeled target.

This expression is the transform pair of (1); moreover, when $R \gg \rho$ and $\alpha=0$, the previous expression becomes a bi- 


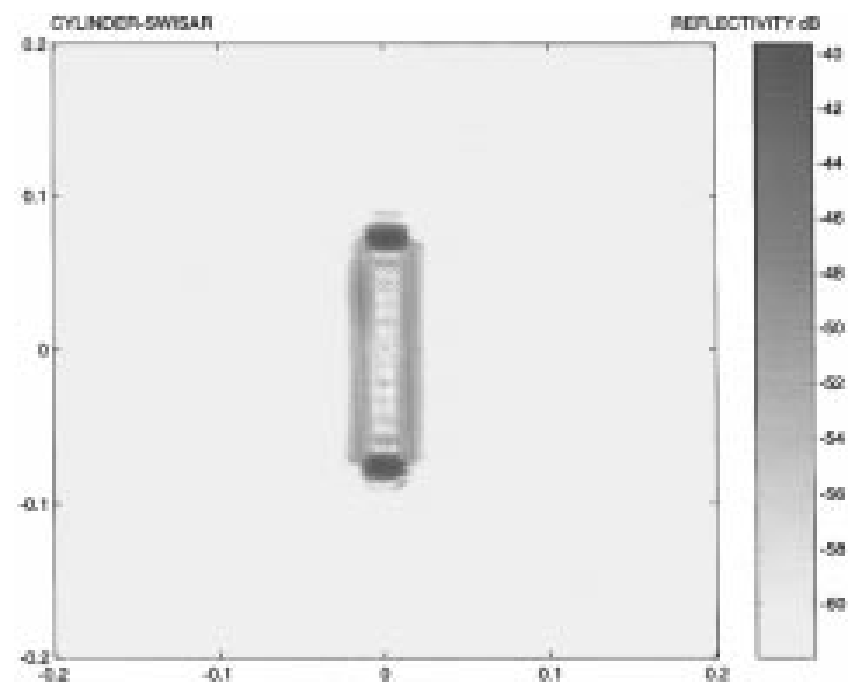

(a)

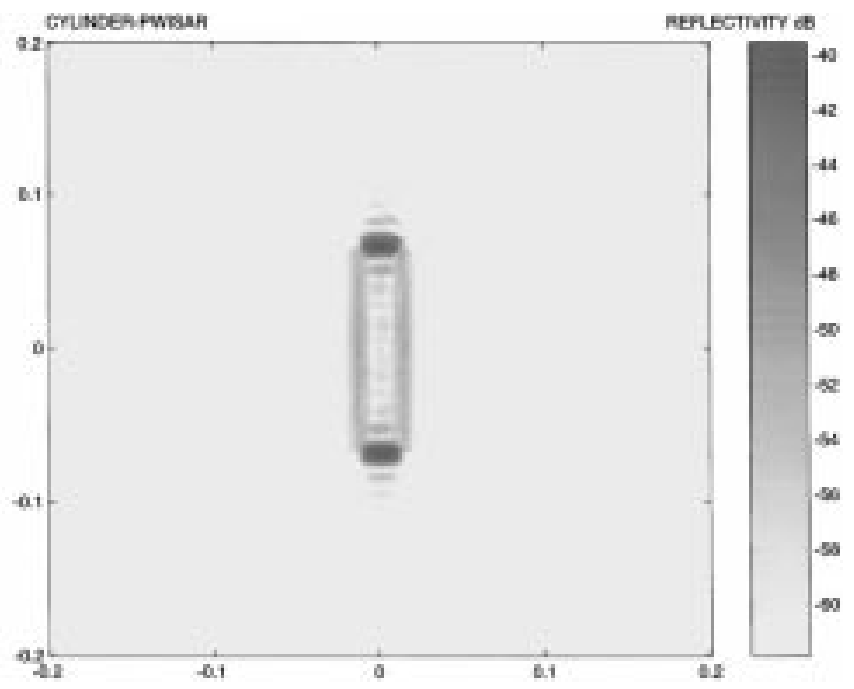

(b)

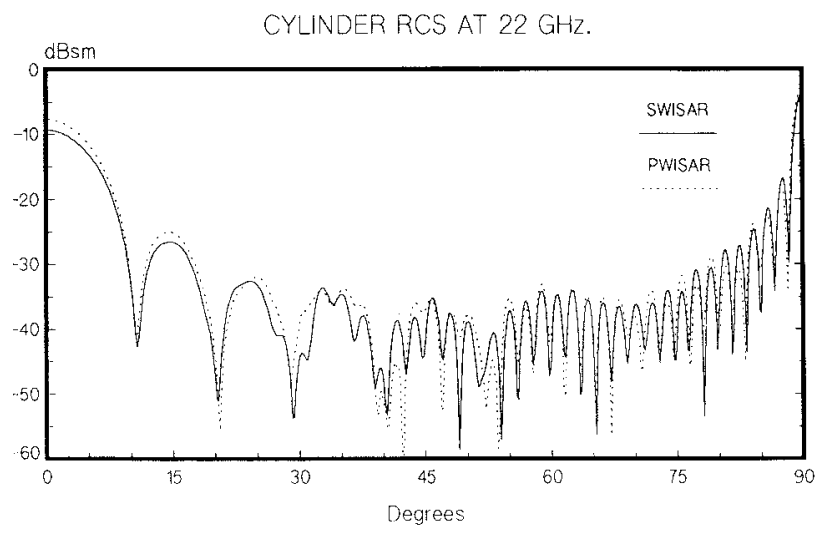

(c)

Fig. 5. (a) Cylinder image obtained with the SWISAR algorithm. (b) Cylinder image obtained with the conventional methods. (c) RCS curves of the cylinder.

dimensional inverse Fourier transform in polar coordinates, which is precisely the conventional ISAR technique to obtain the reflectivity image from the scattering fields produced with a plane wavefront [1].

\section{RCS CALCULATION}

It is well known [1] that if a target formed by independent and nondirectional scatterers is illuminated with a plane wavefront in a monostatic geometry, the coherent superposition of fields scattered from each element corresponds to the total scattered field of the target at each frequency and scan angle

$$
\begin{aligned}
& \sigma(f, \theta) \\
& \quad=e^{-j 2 \frac{2 \pi}{\lambda} R} \int_{-\infty}^{\infty} \int_{-\infty}^{\infty} \psi(x, y) e^{j 2 \frac{2 \pi}{\lambda}(x \cos \theta+y \sin \theta)} d x d y .
\end{aligned}
$$

Since this value is closely related to the RCS one, from now on it will be called indistinctly RCS. In fact, we have

$$
\operatorname{RCS}(f, \theta)=|\sigma(f, \theta)|^{2} .
$$

Apart from constants, this expression is identical to a bi-dimensional Fourier transform of the reflectivity image expressed in Cartesian coordinates. The RCS can, therefore, be determined as a sample of the bi-dimensional Fourier spectrum of the reflectivity image

$$
\begin{aligned}
\Psi\left(f_{x}, f_{y}\right) & =\int_{-\infty}^{\infty} \int_{-\infty}^{\infty} \psi(x, y) \cdot e^{-j 2 \pi\left(x \cdot f_{x}+y \cdot f_{y}\right)} d x d y \\
& \Rightarrow \sigma(f, \theta)=\left.\Psi\left(f_{x}, f_{y}\right)\right|_{f_{x}=-\frac{2}{\lambda} \cos \theta, f_{y}=-\frac{2}{\lambda} \sin \theta} .
\end{aligned}
$$

The numerical values of the RCS parameter finally obtained are affected by a constant that depends on the frequency and azimuth ranges of the measurement and other parameters. Thus, a calibration of the results will be needed in order to obtain absolute RCS values.

\section{ALGORITHM IMPLEMENTATION}

Equation (2) is computed using a circular convolution algorithm over the azimuth angle: since $\xi$ is a function of $(\phi-\theta)$ and $E_{s}$ is a function of only $(\theta)$, the integration in $\theta$ can be interpreted as a circular convolution which can be easily computed as a product in the Fourier spectral domain. 


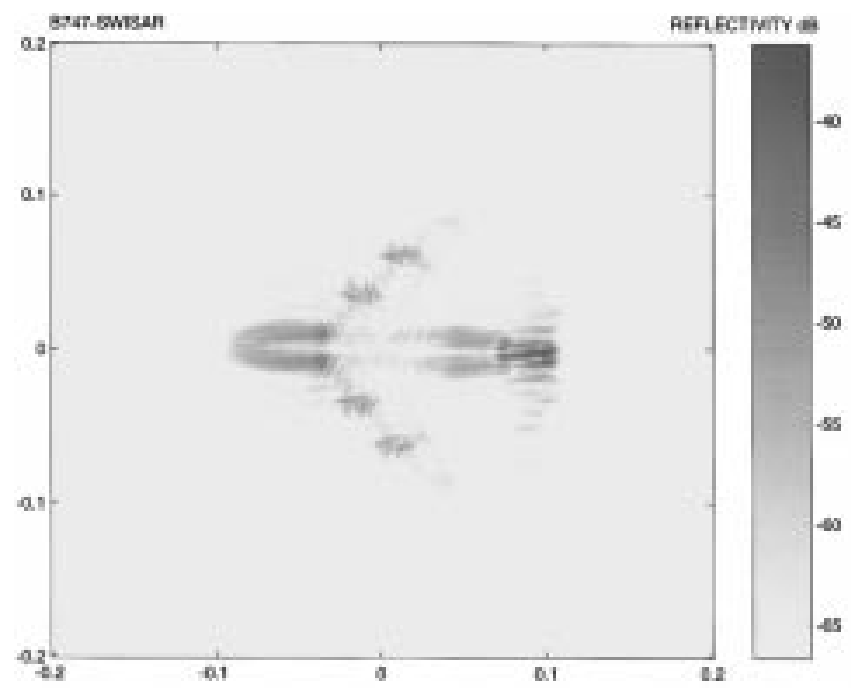

(a)

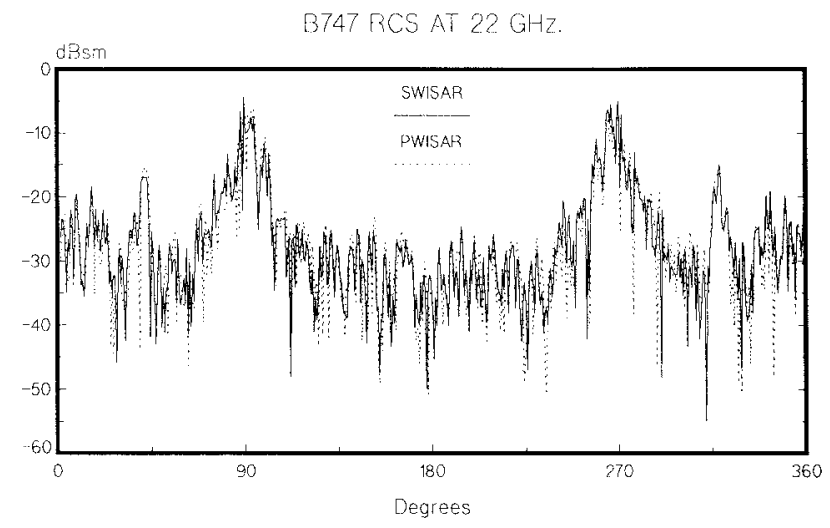

(c)

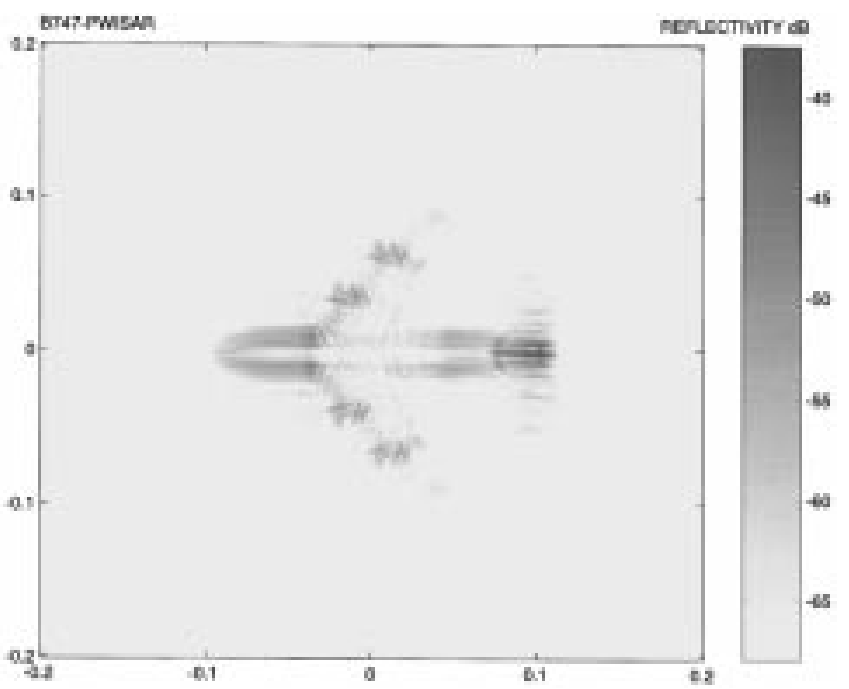

(b)

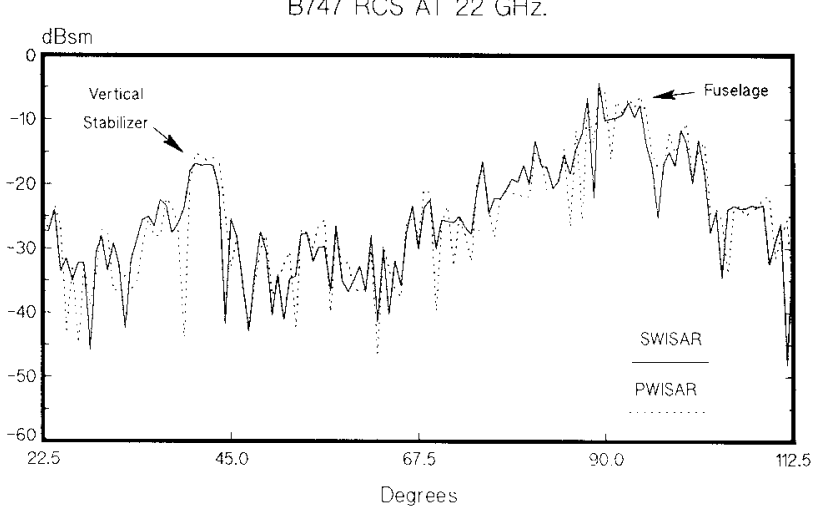

(d)

Fig. 6. (a) B747 image obtained with the SWISAR algorithm. (b) B747 image obtained with the conventional methods. (c) RCS curves of the B747. (d) RCS curves of the B747 (detail).

A similar approach has been applied for plane waves [5]:

$$
\begin{aligned}
\psi(\rho, \phi) & =\int_{0}^{\infty} \int_{0}^{2 \pi} E_{s}(f, \theta) \cdot \xi(f, \rho, \phi-\theta) d f d \theta \\
& =\int_{0}^{\infty} E_{s}(f, \theta) * \xi(f, \rho, \phi) d f \\
\psi(\rho, \phi) & =\sum_{f} E_{s}(f, \theta) * \xi(f, \rho, \phi) \\
& =\sum_{f} \operatorname{FFT}_{\phi}\left[\operatorname{FFT}_{\phi}\left[E_{s}(f, \theta)\right] \cdot \operatorname{FFT}_{\phi}[\xi(f, \rho, \phi)]\right] .
\end{aligned}
$$

The required normalization factors can be computed using the advantage that $\xi(f, \rho, \phi)$ for a given system is constant; therefore, storing its fast Fourier transform (FFT) values on disk reduces the computation time.

Having obtained the reflectivity image, the following three steps shown in Fig. 2 are required to obtain the RCS values:

- Bi-dimensional interpolation: $\psi(x, y)=\psi(\rho, \phi)$ $\left.\right|_{x=\rho \cos \theta, y=\rho \sin \theta}$

- Bi-dimensional Fourier transform: $\Psi\left(f_{x}, f_{y}\right)=\mathrm{FFT}_{2 D}$ $[\psi(x, y)]$

- Bi-dimensional interpolation: $\sigma(f, \theta)=\Psi\left(f_{x}, f_{y}\right)$ $\left.\right|_{f_{x}}=-\frac{2}{\lambda} \cos \theta, f_{y}=-\frac{2}{\lambda} \sin \theta$.
Having obtained the RCS parameter, we adress the calibration problem. The reference used to obtain absolute values is the point spread function (PSF), which is simply a point scatterer of unitary RCS located at the origin of coordinates, which can also be interpreted as the bi-dimensional impulse response of the imaging procedure.

The calibration uses the observation that the scattered fields and the recovered RCS of the PSF have to be one. The calibration consists of dividing, for each frequency and scan angle, the calculated target RCS by the one obtained being the PSF the input of the process. If the object were the PSF, therefore, resulting value would be correctly one. Fig. 3 shows the global procedure followed.

\section{AdDitional AdVANTAges}

This new algorithm, called spherical wave ISAR SWISAR, has additional advantages apart from simplicity and accuracy: owing to the focusing operator $\xi$, effects as the nonisotropic radiation patterns of the antennas and the existence of a bistatic angle can be completely incorporated to the algorithm with no additional complication as indicated by (2). Using nonisotropic antennas it is possible to focus the power on the target and, in addition, reduce the undesired reflections on the anechoic 
chamber and the coupling between the emitter and receiver antennas.

On the other hand, when monostatic measurements are required, two close in-space antennas are often used in a slightly bistatic geometry; this gives approximated results according to the bistatic equivalence theorem. The SWISAR approach avoids this approximation because the formulation accounts for bistatic geometries.

\section{RESULTS}

The procedure has been tested with numerical simulations and experimental results and it shows that the reflectivity images and the RCS curves are quite similar to those obtained with a plane wavefront, both in shape and in absolute values.

Numerical simulations have been used for a preliminary validation of the new method. Fig. 4 shows the results corresponding to a radar target modeled by a number of point scatterers on a wide amplitude range [Fig. 4(a)]: the reconstructed reflectivity image [Fig. 4(b)] shows that the dynamic range of the SWISAR algorithm is around $40 \mathrm{~dB}$, while the good correlation between the theoretical and calculated RCS curves [Fig. 4(c)] leads to the validation of the new algorithm for targets formed by independent point-like scatterers. Some targets cannot be adequately modeled by a group of independent scatterers. Although this assumption is always adopted in ISAR formulation (either with plane or with spherical wave illumination methods), multiple scattering mechanisms like double reflections do appear in the radar reflectivity image [1]. Since the RCS values are obtained from that image, multiple scattering contributions will be also present in the resulting RCS.

This point has been assessed experimentally in an anechoic chamber using metallic cylinders and plane models as targets. Both spherical and plane waves have been used for the illumination. The instrumentation is based on the HP8510 network analyzer, which allows a basic calibration with a reference target and time gating of the quiet zone in order to avoid undesired reflections. The measurements have been made in the K-band (18-26 GHz) over the entire angular range $\left(360^{\circ}\right)$ with the antennas using in the horizontal-horizontal (HH) polarization. The radiation patterns of the antennas have been characterized and incorporated to the algorithm. The distances from the antennas to the origin have been $R_{1}=$ $R_{2}=1.3 \mathrm{~m}$ and the bistatic angle between them $\beta=3.3^{\circ}$.

From numerical simulations it has been found that the minimum distance between target and antennas is two-dimensional (2-D), D being the maximum azimuth dimension of the target; shorter distances will lead to high-power differences between the center of the target and its periphery that could surpass the dynamic range of the system and amplify the noise effects.

Fig. 5 shows the results obtained from a cylinder $228 \mathrm{~mm}$ high and $45 \mathrm{~mm}$ wide. Because the cylinder is a canonical target widely studied, the reflectivity image and RCS response are well known. The images reconstructed with the SWISAR algorithm and the conventional methods are presented in Fig. 5(a) and (b) and the superposition of the RCS curves at $22 \mathrm{GHz}$ obtained with both procedures are shown in Fig. 5(c).

Fig. 6 shows the results for a complex radar target consisting of a metallized Boeing 747 model (1:200 scale). Similarly, the reflectivity images reconstructed from spherical and plane wavefront illuminations are shown in Fig. 6(a) and (b) and the superimposed RCS curves at $22 \mathrm{GHz}$ are shown in Fig. 6(c). The region of these curves corresponding to the higher reflectivity areas (fuselage and vertical stabilizer) are marked to indicate the high correlation between the two curves [Fig. 6(d)].

\section{CONCLUSION}

While microwave imaging, object recognition, and radar target modeling are gaining increasing interest and application, a serious practical problem appears in the creation of the desired plane wavefront needed to carry out the RCS measurements. The problem is especially acute in high-frequency reconstructions of large objects.

An alternative RCS measurement procedure based on spherical wavefront near-field scattering measurements has been presented, which is much easier to obtain.

The measurement distance can be reduced to twice the maximum azimuth dimension of the target and although farfield conditions must be still satisfied in the vertical dimension, most of the radar targets fit the required geometry quite well.

Apart from that, the illumination frequency can be increased without any technical problem while producing the spherical wavefront. Deviations from an ideal spherical wave can be taken in account in the algorithm by characterizing the radiation patterns of the antennas.

This technique is being extended now to three-dimensional scatterers for cylindrical and spherical scanning geometries [6].

\section{ACKNOWLEDGMENT}

The authors would like to thank the anonymous reviewers for their help in improving the paper.

\section{REFERENCES}

[1] D. L. Mensa, High Resolution Radar Imaging. Norwood, MA: Artech House, 1982.

[2] N. C. Curie, Radar Reflectivity Measurement. Norwood, MA: Artech House, 1989.

[3] A. Broquetas, L. Jofre, and A. Cardama, "A near field spherical wave inverse synthetic aperture radar technique," in IEEE AP-S Symp. Dig., Chicago, IL, July 1992, vol. 2, pp. 1114-1117.

[4] J. Palau, A. Broquetas, L. Jofre, and A. Cardama, "Spherical wave nearfield illumination radar cross section measurement," in Proc. PIERS, Jet Propulsion Lab., Pasadena, CA, July 1993, p. 230.

[5] D. L. Mensa, S. Halevy, and O. Wade, "Coherent doppler tomography for microwave imaging," Proc. IEEE, vol. 71, pp. 254-261, Feb. 1983.

[6] X. Anguela and A. Broquetas, "A near-field 3D radar imaging technique," in 23rd Eur. Microwave Conf. Proc., Madrid, Spain, Sept. 1993, pp. $377-379$.

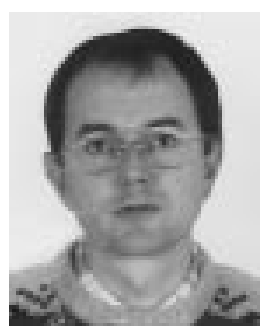

Antoni Broquetas (S'84-M'90) was born in Barcelona, Spain, in 1959. He received the Ing. and Doctor Ing. degrees in telecommunication engineering, both from the Universitat Politïcnica de Catalunya (UPC), Barcelona, Spain, in 1985 and 1989 , respectively.

In 1986, he was a Research Assistant at the Portsmouth Polytechnic, U.K., involved in propagation studies. In 1987 he joined the Department of Signal Theory and Communications of the School of Telecommunication Engineering of the UPC. He is currently an Associate Professor at UPC, involved in research on radar imaging and remote sensing. 


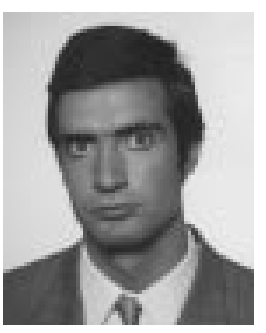

Josep Palau was born in Lleida, Spain, in 1968. He received the Ing. degree in telecomunicacion from the Universitat Politïcnica de Catalunya (UPC), Barcelona, Spain, in 1993

Since 1994, he has been with Andersen Consulting, Barcelona, Spain.

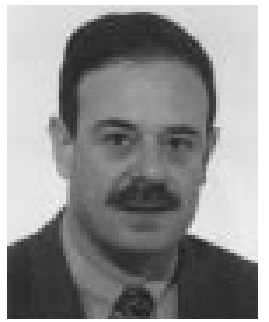

Luis Jofre (S'79-M'83) was born in Mataró, Spain in 1956. He received the Ing. and Doctor Ing. degrees in telecommunication engineering, both from the Universitat Politicnica de Catalunya (UPC), Barcelona, Spain, in 1978 and 1982, respectively.

In 1978, he was a Research Assistant in the Electrophysics Group at UPC, where he worked on the analysis and near-field measurement of antennas. In 1981 he joined the Ecole Supèrieure d'Electricitè, Paris, France, where he was involved in microwave imaging techniques for biomedical applications. From 1986 to 1987 he was a Visiting Fullbright Scholar at the Georgia Institute of Technology, Atlanta, working on antenna measurement and electromagnetic imaging. He is currently a Professor of the Telecommunications Engineering School and Vice-Rector for Academic Planning at UPC. He is engaged in research involving antennas and electromagnetic scattering and imaging.

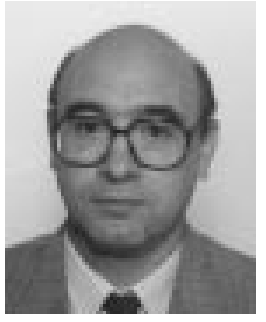

Angel Cardama (S'67-M'73) was born in Santiago, Spain, on May 13, 1944. He received the Ing. de telecomunicación degree from the Universidad Politécnica de Madrid, Spain, in 1968, and the M.Sc. and Ph.D. degrees in electrical engineering from Brown University, Providence, RI, in 1979 and 1973, respectively.

In 1972, he joined the faculty of the E.T.S.I. de Telecomunicación at the Polytecnic University of Catalonia (UPC), Barcelona, Spain, where he holds the position of Professor. His research interests involve propagation in optical fibers, high-frequency apertures and array antennas, and near-field scanning systems to the design of microwave imaging systems and radar antennas. 